\title{
Dominance Measuring Method Performance under Incomplete Information about Weights
}

\author{
ALFONSO MATEOS, ANTONIO JIMÉNEZ and JOSÉ F. BLANCO \\ Departamento de Inteligencia Artificial, Universidad Politécnica de Madrid, Boadilla del Monte, \\ Madrid, Spain
}

\section{INTRODUCTION}

In multi-attribute utility theory (MAUT), the functional form of the multi-attribute utility function differs subject to a variety of independence conditions, see Keeney and Raiffa (1976). The additive model is considered to be a valid approach in most practical situations for the reasons described in Raiffa (1982) and Stewart (1996). The functional form of this model is

$$
u\left(A_{l}\right)=\sum_{i} w_{i} u_{i}\left(x_{i}^{l}\right),
$$

where $x_{i}^{l}$ is the performance of alternative $A_{l}$ in the attribute $X_{i}, u_{i}\left(x_{i}^{l}\right)$ is the utility associated with the aforementioned performance, for $u_{i}(\cdot)$ is the corresponding component utility function representing the decision maker's (DM) preferences over the possible attribute performances and $w_{i}$ are the weights representing the relative importance of each attribute. Note that $\sum_{i} w_{i}=1$.
However, complex decision-making problems are usually plagued with uncertainty. Additionally, it is often not easy to elicit precise values for the scaling weights. They are often described within prescribed bounds or as just satisfying certain ordinal relations. Different authors refer to this situation as decisionmaking with imprecise information, with incomplete information or with partial information.

Several reasons are given in the literature to justify why a DM may wish to provide incomplete information (Weber, 1987; Sarabando and Dias, 2010). Regarding weights, the DM may find it difficult to compare criteria or may not want to reveal his or her preferences in public. Moreover, the decision could be taken in a group decision-making situation, where incomplete information, such as weight intervals, is usually derived from a negotiation process (Jiménez et al., 2005; Mateos et al., 2006).

Many work on MAUT has dealt with incomplete information. Sage and White (1984) proposed the model of imprecisely specified MAUT, where preference information about both weights and utilities is assumed not to be precise. Malakooti (2000) suggested a new efficient algorithm for ranking alternatives when there is incomplete information about the preferences and the value of the alternatives. This involves solving 
a single mathematical programming problem many times. Ahn (2003) extended Malakooti's work.

Eum et al. (2001) provided linear programming characterizations of dominance and potential optimality for decision alternatives when information about performances and/or weights is incomplete, extended the approach to hierarchical structures (Lee et al., 2002) and developed the concepts of weak potential optimality and strong potential optimality (Park, 2004). More recently, Mateos et al. (2007) considered the more general case where imprecision, described by means of fixed bounds, appears in alternative performances, as well as in weights and utilities.

Sarabando and Dias (2010) gave a brief overview of approaches proposed by different authors within the MAUT and MAVT (multi-attribute value theory) framework to deal with incomplete information.

Stochastic multicriteria acceptability analysis (SMAA) was developed for support in discrete group decision-making problems, where there is no weight information (Lahdelma and Salminen, 1998), and is widely used. This situation was also considered by Bana e Costa (1986), Charnetski and Soland (1978), Nijkamp et al. (1990) and Voogd (1983). SMAA explores the weight space to describe the ratings that would make each alternative the preferred one and computes acceptability indices. Acceptability indices measure the variety of different preferences ranking each alternative best. This information can be used to classify the alternatives as more or less acceptable and unacceptable. However, SMAA ignores information about the other ranks. This can make it difficult to identify good compromise alternatives, particularly when the acceptability is split between extreme alternatives; each of which is ranked best according to a few weights but worst by other weights. The potential compromise alternatives then are the ones that yield a good, but not necessarily the best, rank across many different weights.

Lahdelma and Salminen (2001) proposed the SMAA-2 method to solve this problem. SMAA-2 extends the analysis to the sets of weight vectors for any rank from best to worst for each decision alternative. The rank acceptabilities can then be combined using the so-called meta weights into holistic acceptability indices, describing the overall acceptability of each alternative.

A new approach for dealing with incomplete information about weights is to use information about each alternative's intensity of dominance, known as dominance measuring methods.

The first dominance measuring method was proposed in Ahn and Park (2008). They computed both dominating and dominated measures from a dominance matrix and then derived a net dominance. This is used as a measure of the strength of preference in the sense that a greater net value is better. They proposed and compared two alternative approaches with surrogate weighting methods and decision rules by means of a simulation study.

In Mateos et al., (2010), we proposed two new dominance measuring methods aimed at overcoming some drawbacks associated with Ahn and Park's methods. The first method is based on dominating and dominated measures computed from the dominance matrix. They are combined into a net dominance value, but they are all computed differently than Ahn and Park's (2008) measures to resolve deficiencies and improve on Ahn and Park's methods. In the second method, alternatives are ranked on the basis of a preference intensity measure. These preference intensity measures are based on the fact that the differences between utilities corresponding to alternatives $A_{k}$ and $A_{l}$ are always within the interval whose lower endpoint is the element located at the $k$ th row and lth column of the dominance matrix and whose upper endpoint is located at the $l$ th row and $k$ th column of the dominance matrix with the sign changed. Moreover, alternative $A_{k}$ is preferred to $A_{l}$ for the positive values in the above interval, whereas alternative $A_{l}$ is preferred to $A_{k}$ for the negative values.

The aforementioned methods considered ordinal relations regarding attribute weights, that is, DMs ranked attributes in descending order of importance. In this paper, we consider different cases with incomplete information about weights. Specifically, we consider weight intervals, weights fitting independent normal probability distributions or weights represented by triangular or trapezoidal fuzzy numbers. Then, a simulation study is performed to compare the proposed methods with the measures reported in Ahn and Park (2008), with classical decision rules and with the SMAA and SMAA-2 methods in the aforementioned situations.

The paper is organized as follows. In Section 2, we examine weight intervals. Pairwise and absolute dominance concepts and derived ranking methods, such as modified classical decision rules and dominance measuring methods, are reviewed, as are the $S M A A$ and SMAA-2 methods. In Section 3, an illustrative example is introduced. Section 4 evaluates and compares the methods in a simulation study and presents the study results. In Section 5, weights fit independent normal probability distributions or are represented by fuzzy numbers. Finally, we outline our conclusions in Section 6. 


\section{RANKING METHODS BASED ON DOMINANCE MEASURES}

Let us consider a group decision-making problem with $n$ attributes $\left(X_{i}, i=1, \ldots, n\right)$ and $m$ alternatives $\left(A_{j}, j=1, \ldots, m\right)$, where incomplete information about input parameters has been incorporated into the decision-making process as follows:

- Alternative performances under uncertainty $\left(x_{i}^{j} \in\left[x_{i}^{j L}, x_{i}^{j U}\right], i=1, \ldots, n ; j=1, \ldots, m\right)$, where $x_{i}^{j L}$ and $x_{i}^{j U}$ are the lower and the upper endpoints of the performance interval of the attribute $X_{i}$ for the alternative $A_{j}$, respectively.

- Imprecision concerning utility function assessment $\left(u_{i}(\cdot) \in\left[u_{i}^{L}(\cdot), u_{i}^{U}(\cdot)\right], i=1, \ldots, n\right)$, where $u_{i}^{L}(\cdot)$ and $u_{i}^{U}(\cdot)$ are the lower and the upper utility functions of the attribute $X_{i}$.

- Imprecision concerning weights, which is represented by weights intervals $\left(w_{i} \in\left[w_{i}^{L}, w_{i}^{U}\right], i=1, \ldots, n\right)$, where $w_{i}^{L}$ and $w_{i}^{U}$ are the lower and the upper endpoints of the weight interval for the attribute $X_{i}$, $i=1, \ldots, n$.

Given two alternatives $A_{k}$ and $A_{l}$, alternative $A_{k}$ dominates $A_{l}$ if $D^{\prime}{ }_{k l} \geq 0, D^{\prime}{ }_{k l}$ being the optimum value of the optimization problem (Puerto et al., 2000),

$$
\begin{aligned}
D_{k l}^{\prime}= & \min \left\{u\left(A_{k}\right)-u\left(A_{l}\right)=\sum_{i=1}^{n} w_{i} u_{i}\left(x_{i}^{k}\right)-\sum_{i=1}^{n} w_{i} u_{i}\left(x_{i}^{l}\right)\right\} \\
& w_{i}^{L} \leq w_{i} \leq w_{i}^{U}, i=1, \ldots, n \\
& x_{i}^{k L} \leq x_{i}^{k} \leq x_{i}^{k U}, i=1, \ldots, n \\
\text { s.t. } \quad & x_{i}^{l L} \leq x_{i}^{l} \leq x_{i}^{l U}, i=1, \ldots, n \\
& u_{i}^{L}\left(x_{i}^{k}\right) \leq u_{i}\left(x_{i}^{k}\right) \leq u_{i}^{U}\left(x_{i}^{k}\right), i=1, \ldots, n \\
& u_{i}^{L}\left(x_{i}^{l}\right) \leq u_{i}\left(x_{i}^{l}\right) \leq u_{i}^{U}\left(x_{i}^{l}\right), i=1, \ldots, n .
\end{aligned}
$$

Examining the objective function, we find that it can be rewritten as $\sum_{i} w_{i}\left[u_{i}\left(x_{i}^{k}\right)-u_{i}\left(x_{j}^{l}\right)\right]$, where $u_{i}\left(x_{i}^{k}\right)$ $u_{i}\left(x_{i}^{l}\right)$ does not depend on weights $w_{i}$. Moreover, if we carefully observe the constraints, we discover that variables $w_{i}$ are independent of the other variables. So, taking into account that weights $w_{i}$ are non-negative, solving problem (1) is equivalent to solving the optimization problem

$$
\begin{aligned}
& D_{k l}^{\prime}=\min \left\{\sum_{i=1}^{n} w_{i} z_{i}^{k l}\right\} \\
& \text { s.t. } w_{i}^{L} \leq w_{i} \leq w_{i}^{U}, i=1, \ldots, n,
\end{aligned}
$$

where $z_{i}^{k l}$ are the optimal values of the optimization problem

$$
\begin{array}{ll}
z_{i}^{k l}= & \min \left\{u_{i}\left(x_{i}^{k}\right)-u_{i}\left(x_{i}^{l}\right)\right\} \\
& x_{i}^{k L} \leq x_{i}^{k} \leq x_{i}^{U}, i=1, \ldots, n \\
& x_{i}^{I L} \leq x_{i}^{l} \leq x_{i}^{I U}, i=1, \ldots, n \\
\text { s.t. } & u_{i}^{L}\left(x_{i}^{k}\right) \leq u_{i}\left(x_{i}^{k}\right) \leq u_{i}^{U}\left(x_{l}^{k}\right), i=1, \ldots, n \\
& u_{i}^{L}\left(x_{i}^{l}\right) \leq u_{i}\left(x_{i}^{l}\right) \leq u_{i}^{U}\left(x_{l}^{l}\right), i=1, \ldots, n .
\end{array}
$$

Problem (3) can be solved depending on what the characteristics of the utility function for attribute $X_{i}$ are (Mateos et al., 2007):

- If the utility function is monotonically increasing, then $z_{i}^{k l}=u_{i}^{L}\left(x_{i}^{k L}\right)-u_{i}^{U}\left(x_{i}^{l U}\right)$.

- If the utility function is monotonically decreasing, then $z_{i}^{k l}=u_{i}^{L}\left(x_{i}^{k U}\right)-u_{i}^{U}\left(x_{i}^{l L}\right)$.

This concept of dominance is called pairwise dominance and leads to the so-called dominance matrix:

$$
D=\left(\begin{array}{lllll}
- & D_{12} & \ldots & D_{1 m-1} & D_{1 m} \\
D_{21} & - & \ldots & D_{2 m-1} & D_{2 m} \\
D_{31} & D_{32} & \ldots & D_{3 m-1} & D_{3 m} \\
& & \ldots & & \\
D_{m 2} & D_{m 2} & \ldots & D_{m m-1} & -
\end{array}\right)
$$

where $D_{k l}=D^{\prime}{ }_{k l} / \sum_{i=1}^{n} w_{i}$ and $D^{\prime}{ }_{k l}$ and $w_{i}$ are the optimum values for the objective function and weights in problem (1), respectively.

Another possibility is to use what is known as absolute dominance (Salo and Hämäläinen, 2001). Absolute dominance considers the following linear optimization problems:

$$
\begin{aligned}
U_{k}= & \max \sum_{i=1}^{n} w_{i} u_{i}\left(x_{i}^{k}\right) \\
& w_{i}^{L} \leq w_{i} \leq w_{i}^{U}, i=1, \ldots, n \\
\text { s.t. } & x_{i}^{k L} \leq x_{i}^{k} \leq x_{i}^{k U}, i=1, \ldots, n \\
& u_{i}^{L}\left(x_{i}^{k}\right) \leq u_{i}\left(x_{i}^{k}\right) \leq u_{i}^{U}\left(x_{i}^{k}\right), i=1, \ldots, n
\end{aligned}
$$


and

$$
\begin{array}{ll}
L_{k}= & \min \sum_{i=1}^{n} w_{i} u_{i}\left(x_{i}^{k}\right) \\
& w_{i}^{L} \leq w_{i} \leq w_{i}^{U}, i=1, \ldots, n \\
\text { s.t. } & x_{i}^{k L} \leq x_{i}^{k} \leq x_{i}^{k U}, i=1, \ldots, n \\
& u_{i}^{L}\left(x_{i}^{k}\right) \leq u_{i}\left(x_{i}^{k}\right) \leq u_{i}^{U}\left(x_{i}^{k}\right), i=1, \ldots, n
\end{array}
$$

Alternative $A_{k}$ absolutely dominates $A_{l}$ if $L_{k}>U_{l}$, that is, the lower bound of $A_{k}$ exceeds the upper bound of $A_{l}$. Note that if $A_{k}$ absolutely dominates $A_{l}$, then $A_{k}$ dominates $A_{l}$, but the reverse does not hold.

Note that this dominance approach often results in almost no prioritization of alternatives or too many non-dominated alternatives (Kirkwood and Corner, 1993). However, pairwise and absolute dominance values can be used to further prioritize competitive alternatives and hence recommend the best alternative and fully rank alternatives.

The following is an example of how these dominance values have been employed to modify three classical decision rules to operate in an imprecise decision-making context (Puerto et al., 2000; Salo and Hämäläinen, 2001):

- maximax rule or optimist rule (OPT): evaluating each alternative for its maximum guaranteed value, that is, $\max \left\{U_{j}, j=1, \ldots, m\right\}$.

- maximin rule or pessimist rule (PES): evaluating each alternative for its minimum guaranteed value, that is, $\max \left\{L_{j}, j=1, \ldots, m\right\}$.

- minimax regret rule (REG): evaluating each alternative for the maximum loss of value with respect to a better alternative, that is, $\min \left\{M R_{k}, k=1, \ldots, m\right\}$, where $M R_{k}$ represents the maximum regret incurred when choosing alternative $l$, that is, $M R_{k}=\max$ $\left\{\max \left\{u\left(A_{l}\right)-u\left(A_{k}\right) \mid \boldsymbol{W}_{k l}\right\} \forall l \neq k\right\}$, where $\boldsymbol{W}_{k l}$ are the constraints of the optimization problem (1).

Although none of these rules ensures that the best ranked alternative is the same as it would be if precise values were elicited for weights, simulations show that the selected alternative is generally one of the best (Sarabando and Dias, 2009).

A new approach is to use information about each alternative's intensity of dominance employing what are known as dominance measuring methods. Ahn and Park (2008) proposed two approaches on the basis of the dominance matrix $D$. In the first approach, denoted by API (Ahn and Park 1), alternatives are ranked according to a dominating measure $\alpha_{k}=\sum_{j=1, j \neq k}^{m} D_{k j}$. The higher this dominating measure is, the more preferred the alternative will be because the sum of the intensity of one alternative dominating the others will also be greater. In the second approach, denoted by AP2 (Ahn and Park 2), alternatives are ranked according to the difference between the dominating measure $\alpha_{k}$ and a dominated measure $\beta_{k}=\sum_{l=1, l \neq k}^{m} D_{l k}$, that is, on the basis of $\alpha_{k}-\beta_{k}$.

A simulation study showed $A P 1$ to be better than $A P 2$. Whereas $A P 1$ involves just adding the paired dominance values in the $k$ th row of $D, A P 2$ considers paired dominance values in both the $k$ th row and the $k$ th column of $D$. The reason why $A P 1$ is better than $A P 2$ is that $A P 2$ uses duplicated information (row and column values). On the other hand, APl only takes into account the dominating measure, leading to a trade-off between positives and negatives. The drawbacks associated with $A P 1$ and $A P 2$ are that $A P 1$ only considers dominating measures (trade-off of positive and negative values), and $A P 2$ duplicates dominated measures.

Two new methods aimed at overcoming these problems were proposed in Mateos et al. (2010). The first one, denoted by DME1 (Dominance Measuring Extension 1), is based on the same idea as Ahn and Park suggested (Ahn and Park, 2008). First, dominating and dominated measures are computed from the paired dominance values $D_{k l}$ and then a net dominance is derived. This net dominance is used as a measure of the strength of preference. DMEl computes the positive and negative dominating measures (step 2) and positive and negative dominated measures (step 4). They are used to compute first a proportion representing how strongly one alternative is preferred to the others (step 3) and second, a proportion representing how intensely one alternative is not preferred to the others (step 5). Finally, DME1 subtracts both proportions (step 6) to compute the intensity of the preference. The ranking of alternatives will be based on this intensity value (step 7).

$D M E 1$ can be implemented as follows:

1. Obtain the paired dominance values $D_{k l}$ and the dominance matrix $D$ as in (4).

2. Compute the dominating measures $\alpha_{k}, \alpha_{k}^{+}$ and $\alpha_{k}^{-}$for each alternative $A_{k}$ :

$$
\alpha_{k}=\sum_{l=1, l \neq k}^{m} D_{k l}, \alpha_{k}{ }^{+}=\sum_{l=1, l \neq k, D_{k l}>0}^{m} D_{k l} \text { and } \alpha_{k}{ }^{-}=\sum_{l=1, l \neq k, D_{k l}<0}^{m} D_{k l}, \forall_{k} .
$$


3. Compute the proportion

$$
p_{k}^{\alpha}=\frac{\alpha_{k}^{+}}{\alpha_{k}^{+}-\alpha_{k}^{-}} .
$$

4. Compute the dominated measures $\beta_{k}, \beta_{k}^{+}$and $\beta_{k}^{-}$ for each alternative $A_{k}$ :

$$
\begin{aligned}
\beta_{\mathbf{k}} & =\sum_{l=1, l \neq k}^{m} D_{l k}, \beta_{k}^{+}=\sum_{l=1, l \neq k, D_{i k}>0}^{m} D_{l k} \text { and } \beta_{k}^{-} \\
& =\sum_{l=1, l \neq k, D_{l k}<0}^{m} D_{l k}, \forall k .
\end{aligned}
$$

5. Compute the proportion

$$
P_{k}^{\beta}=\frac{\beta_{k}^{+}}{\beta_{k}^{+}-\beta_{k}^{-}} .
$$

6. Calculate the preference intensity value $P_{k}$ for each alternative $A_{k}$ :

$$
P_{k}=P_{k}^{\alpha}-P_{k}^{\beta}, k=1, \ldots, m .
$$

7. Rank alternatives according to the $P_{k}$ values, where the best (rank 1) is the alternative for which $P_{k}$ is a maximum and the worst (rank $m$ ) is the alternative for which $P_{k}$ is the minimum.

The drawback of the DME1 method is that when the dominance matrix $D$ contains all negative elements, that is, when all the alternatives are non-dominated, the algorithm is unable to rank the alternatives because all $P_{k}$ are equal to 0 . The properties of matrix $D$ were analysed in an attempt to overcome this problem, and we observed that the difference between the utilities corresponding to $A_{k}$ and $A_{l}, \sum_{i=1}^{n} w_{i} u_{i}\left(x_{i}^{k}\right)-\sum_{i=1}^{n} w_{i} u_{i}\left(x_{i}^{l}\right)$ in $\boldsymbol{W}_{k l}$, is always within $\left[D_{k l},-D_{l k}\right]$, as demonstrated in Mateos et al. (2010). Thus,

- If $-D_{l k} \leq 0 \Leftrightarrow D_{k l}<0$ and $D_{l k} \geq 0 \Leftrightarrow$ alternative $A_{l}$ dominates $A_{k} \Rightarrow$ the intensity with which $A_{k}$ is preferred to $A_{l}$ is 0 , that is, $P I_{k l}=0$.

- If $D_{k l} \geq 0 \Leftrightarrow D_{k l} \geq 0$ and $D_{l k}<0 \Leftrightarrow$ alternative $A_{k}$ dominates $A_{l} \Rightarrow$ the intensity with which $A_{k}$ is preferred to $A_{l}$ is 1 , that is, $P I_{k l}=1$.

- If $D_{k l}<0$ and $D_{l k}<0$, then interval $\left[D_{k l},-D_{l k}\right]$ will contain a positive subinterval with positive values in which alternative $A_{k}$ is preferred to $A_{l}$ and a negative subinterval in which alternative $A_{l}$ is preferred to $A_{k}$. Thus, the intensity with which $A_{k}$ is preferred to $A_{l}$ is the proportion of the positive subinterval over the whole $\left[D_{k l},-D_{l k}\right]$.

On the basis of this idea, a second method was proposed in Mateos et al. (2010), denoted by DME2 (Dominance Measuring Extension 2). In DME2, paired dominance values $D_{k l}$ are first transformed into preference intensities $P I_{k l}$ (step 2) depending on the preference among alternatives $A_{k}$ and $A_{l}$. Then, a preference intensity measure $\left(P I M_{k}\right)$ is derived for each alternative $A_{k}$ (step 3 ) as the sum of the preference intensities of alternative $A_{k}$ regarding the other alternatives. This is used as the measure of the strength of preference.

Dominance measuring extension 2 can be implemented as follows:

1. Compute dominance matrix $D(4)$ from the paired dominance values $D^{\prime}{ }_{k l}(1)$.

2. If $D_{k l} \geq 0$, then alternative $A_{k}$ is preferred to alternative $A_{l}$, that is, the intensity with which alternative $A_{k}$ is preferred to $A_{l}$ is $1, P I_{k l}=1$.

Else $\left(D_{k l}<0\right)$,

- If $D_{l k} \geq 0$, then alternative $A_{l}$ dominates alternative $A_{k}$; therefore, the intensity with which alternative $A_{k}$ is preferred to $A_{l}$ is 0 , that is, $P I_{k l}=0$.

- Else, note that alternative $A_{1}$ is preferred to alternative $A_{k}$ for those values in $\boldsymbol{W}_{k l}$ that satisfy $D_{k l} \leq \sum_{i=1}^{n} w_{i} u_{i}\left(x_{i}^{k}\right)-\sum_{i=1}^{n} w_{i} u_{i}\left(x_{i}^{l}\right) \leq 0$, and $A_{k}$ is preferred to $A_{l}$ for those values in $W_{k l}$ that satisfy $0 \leq \sum_{i=1}^{n} w_{i} u_{i}\left(x_{l}^{k}\right)-\sum_{i=1}^{\mathrm{n}} w_{i} u_{i}\left(x_{i}^{l}\right) \leq-D_{l k} \Rightarrow$ the intensity $A_{k}$ is preferred to $A_{l}$ is

$$
P I_{k l}=\frac{-D_{l k}}{-D_{l k}-D_{k l}}
$$

3. Compute a preference intensity measure for each alternative $A_{k}$

$$
P I M_{\mathbf{k}}=\sum_{l=1, l \neq k}^{m} P I_{k l} \text {. }
$$

4. Rank alternatives according to the $P I M$ values, where the best (rank 1) is the alternative with greatest PIM and the worst is the alternative with the least PIM. 
As mentioned before, $D M E 1$ and $D M E 2$, same with $A P 1$ and $A P 2$, considered ordinal relations regarding attribute weights, that is, DMs ranked attributes in descending order of importance. For this scenario, Monte Carlo simulation techniques were carried out to analyse their performance and to compare them with other approaches, such as surrogate weighting methods and adapted classical decision rules (Ahn and Park, 2008; Mateos et al., 2010).

The results showed that DME2 performs better in terms of the identification of the best alternative and the overall ranking of alternatives than other dominance measuring methods proposed by different authors. Also, DME2 outperforms the adaptation of classical decision rules and comes quite close to the Rank-Order centroid $R O C$ method, which was identified as the best approach.

In Section 4, the results of a new computational study based on Monte Carlo simulation techniques are shown for a scenario where there are weight intervals rather than ordinal relations. Surrogate weighting methods cannot be used for this purpose, so the AP1 and DME2 methods are analysed and compared with modified decision rules and with SMAA and SMAA-2 methods, which are briefly described in the succeeding text.

Stochastic multicriteria acceptability analysis (Lahdelma and Salminen, 1998) is widely used for situations where neither criteria values nor weights are precisely known. Uncertain or imprecise criteria values are represented by stochastic variables $\varepsilon_{i j}$ with assumed or estimated joint probability distribution and density function $f(\varepsilon)$ in the space $X$. Similarly, the DM's unknown or partially known preferences are represented by a weight distribution with density function $f(w)$ in the set of feasible weights $\boldsymbol{W}$. Total lack of knowledge about weights is represented in 'Bayesian' spirit by a uniform weight distribution in $\boldsymbol{W}$. The distribution has density function $f(w)=\frac{1}{\operatorname{vol}(W)}$.

The SMAA method determines for each alternative the set of favourable weights $\boldsymbol{W}_{i}(\varepsilon)=\left\{w \in \boldsymbol{W}: u\left(\varepsilon_{i}, w\right) \geq\right.$ $\left.u\left(\varepsilon_{k}, w\right), \forall k\right\}$. Then, the acceptability index is used to classify the alternatives as more or less acceptable $\left(a_{i}>0\right)$ and unacceptable ( $a_{i}$ zero or near zero), where

$$
a_{i}=\int_{X} f(\varepsilon) \int_{\boldsymbol{W}_{\mathrm{i}}(\varepsilon)} f(w) d w d \varepsilon
$$

In some cases, some amount of bias may occur in the SMAA acceptability indices: a) the scaling of the criteria affects the acceptability indices; b) if the assumed weight distribution does not represent the DMs' preference accurately, classification based on acceptability indices should be limited to excluding alternatives with zero or near-zero acceptability; and c) SMAA ignores information about the other ranks. For this reason, the SMAA-2 method (Lahdelma and Salminen, 2001) was developed. SMAA-2 extends the acceptability analysis to the sets of weight vectors for each rank from best to worst for each decision alternative.

The rank acceptability index $a_{i}^{r}$ is then defined as the expected volume of the set of favourable rank weights. The rank acceptability index is a measure of the variety of different ratings ranking alternative $A_{i}$ as $r$,

$$
a_{i}^{r}=\int_{X} f(\varepsilon) \int_{\boldsymbol{W}_{i}^{r}(\varepsilon)} f(w) d w d \varepsilon
$$

where $\boldsymbol{W}_{i}^{r}(\varepsilon)=\left\{w \in \boldsymbol{W}: \operatorname{rank}\left(\varepsilon_{i}, w\right)=r\right\}$ with rank $\left(\varepsilon_{i}, w\right)=1+\sum_{k} \rho\left(u\left(\varepsilon_{k}, w\right)>u\left(\varepsilon_{k}, w\right)\right)$ and $\rho($ true $)=1$ and $\rho($ false $)=0$. The problem of comparing the alternatives in terms of their rank acceptabilities can be seen as a 'second-order' multicriteria decision problem. For this reason, Lahdelma and Salminen (2001) proposed a complementary approach of combining the rank acceptabilities into holistic acceptability indices $a_{i}$ for each alternative using

$$
a_{i}=\sum_{r} \alpha^{r} a_{i}^{r},
$$

where $\alpha^{r}=\left(\sum_{i=r}^{m} 1 / i\right) /\left(\sum_{i=1}^{m} 1 / i\right)$ are centroid weights.

\section{AN ILLUSTRATIVE EXAMPLE}

To illustrate the methods introduced in the previous section, we consider the data from a decision-making problem consisting of the construction of a new general cargo harbour in Helsinki, Finland (Hokkanen et al., 1999; Lahdelma and Salminen, 2001). Table I shows the criteria performances for $m=13$ alternatives (where I and II are navigation channels; A, B and C represent roads; and 1,2, 3 and 4 correspond to railway connections) and $n=11$ attributes.

Uniform probability distributions for criteria performances in the intervals $\left[x_{i j}-\Delta_{i j}, x_{i j}+\Delta_{i j}\right]$ are used. For $\Delta_{i j}, 10 \%$ of the interval between the highest and lowest performance for each criteria is used (i.e. $\left.\Delta_{i j}=\left(x_{j}^{\max }-x_{j}^{\min }\right) / 10\right)$. The scaling problem is also outlined here; the criteria performances are scaled by using best and worst criteria values, that is, $u_{i j}=\left(x_{i j}-x_{j}^{\text {worst }}\right) /\left(x_{j}^{\text {best }}-x_{j}^{\text {worst }}\right)$. 
Table I. Criteria performances

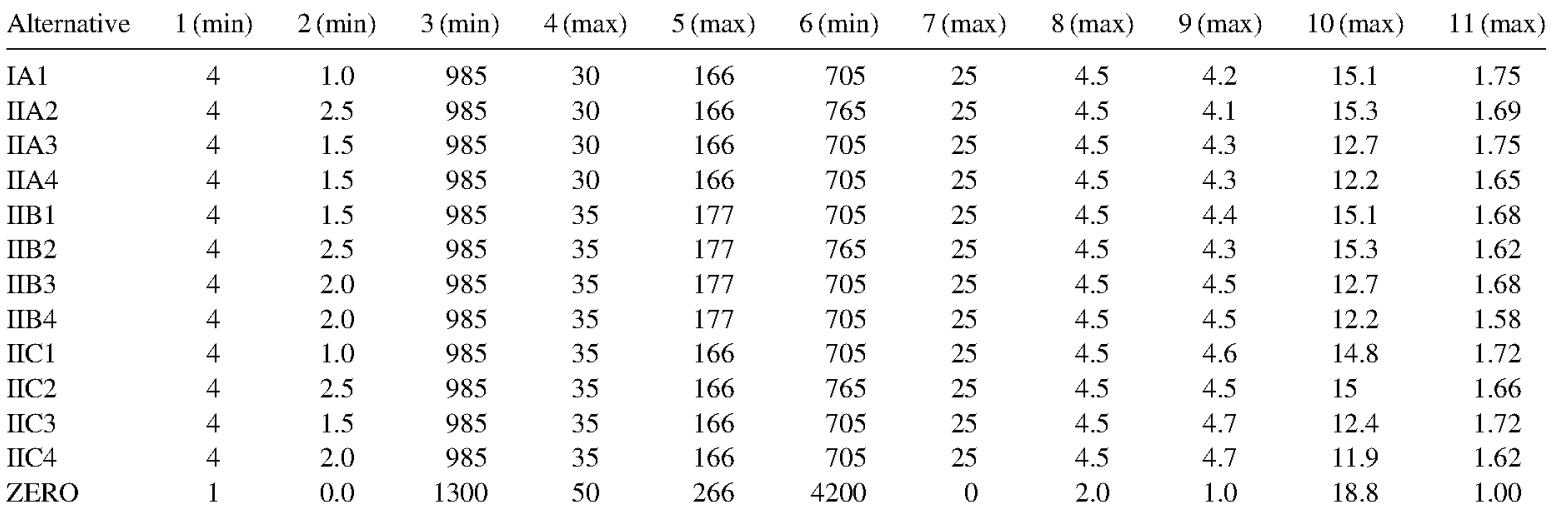

Table II shows the dominance matrix. Note that, in this example, a simple operation solves the optimization problem (2). For example,

$$
\begin{gathered}
D_{12} \min \{0-0.1 \hat{6}, 0.5-0.1 \hat{6}, 0.57-1,0-0.1 \hat{6}, 0 \\
-0.1 \hat{6}, 0.8 \hat{3}-0.99,0.8 \hat{3}-1,0.8 \hat{3}-1,0.72 \\
-0.86,0.45-0.59,08 \hat{3}-0.9 \hat{3}\}=-0.43 .
\end{gathered}
$$

Rows 1 and 2 of Table III show the results output by Ahn and Park's method and the corresponding ranking, respectively. Each element in the first row is computed by adding the values of the corresponding row in Table II.

To apply the DME2 method, we first have to compute a preference intensity matrix from the dominance matrix and then the preference intensity measure for each alternative. Table IV shows the preference intensity matrix, whereas Table $\mathrm{V}$ lists the preference intensity measures for each alternative and the resulting ranking.

Note that the first value in Table IV is computed as follows: $P_{k l}=\frac{0.6}{0.6+0.43}=0.607$. Values shown in Table $\mathrm{V}$ are computed by adding the values in the corresponding row of Table IV (the first row is used for the first value, and so on).

Finally, Table VI shows the results output by the $S M A A$ and SMAA-2 methods, see Lahdelma and Salminen (2001).

Figure 1 illustrates the alternative rankings included in Tables III and V together with the results of the SMAA and SMAA-2 methods.

Looking at Figure 1, we find that

- The best alternative is IIC3 for all four methods, whereas the second-ranked is the same for the
DME2, SMAA and SMAA-2 methods (IIC1), but this alternative is ranked as seventh by the $A P I$ method.

- The best two and the worst three alternatives are the same for the DME2 method, analysing the set of favourable weights (SMAA and SMAA-2 methods). This similarity is stronger for the $D M E 2$ and SMAA-2 methods, which also output the same alternatives for the third, fourth, fifth and sixth rankings, whereas IIA4 and IIB1 just switch positions (seventh and eighth). Looking at Tables V and VI, we find that the difference between IIA4 and IIB1 is minimum, although IIA4 was possibly a little better. The same applies to the tenth-ranked and eleventh-ranked alternatives.

- The main difference between the ranking output by the SMAA method and DME2 and SMAA-2 methods is that alternative ZERO is ranked fourth by $S M A A$, whereas the DME2 and SMAA-2 methods rank it as the ninth and tenth, respectively.

- In any case, the results output by the DME2, SMAA and SMAA-2 methods are very similar.

Generally, $D M E 2$ is very easy to apply and computationally efficient. On the other hand, the SMAA-2 method shows more information. In our opinion, the $D M E 2$ approach could be used to output the ranking of alternatives, and the SMAA-2 method could be used to study the robustness of this ranking.

\section{COMPUTATIONAL STUDY}

We propose to conduct a simulation study of the dominance measuring methods described in the previous 

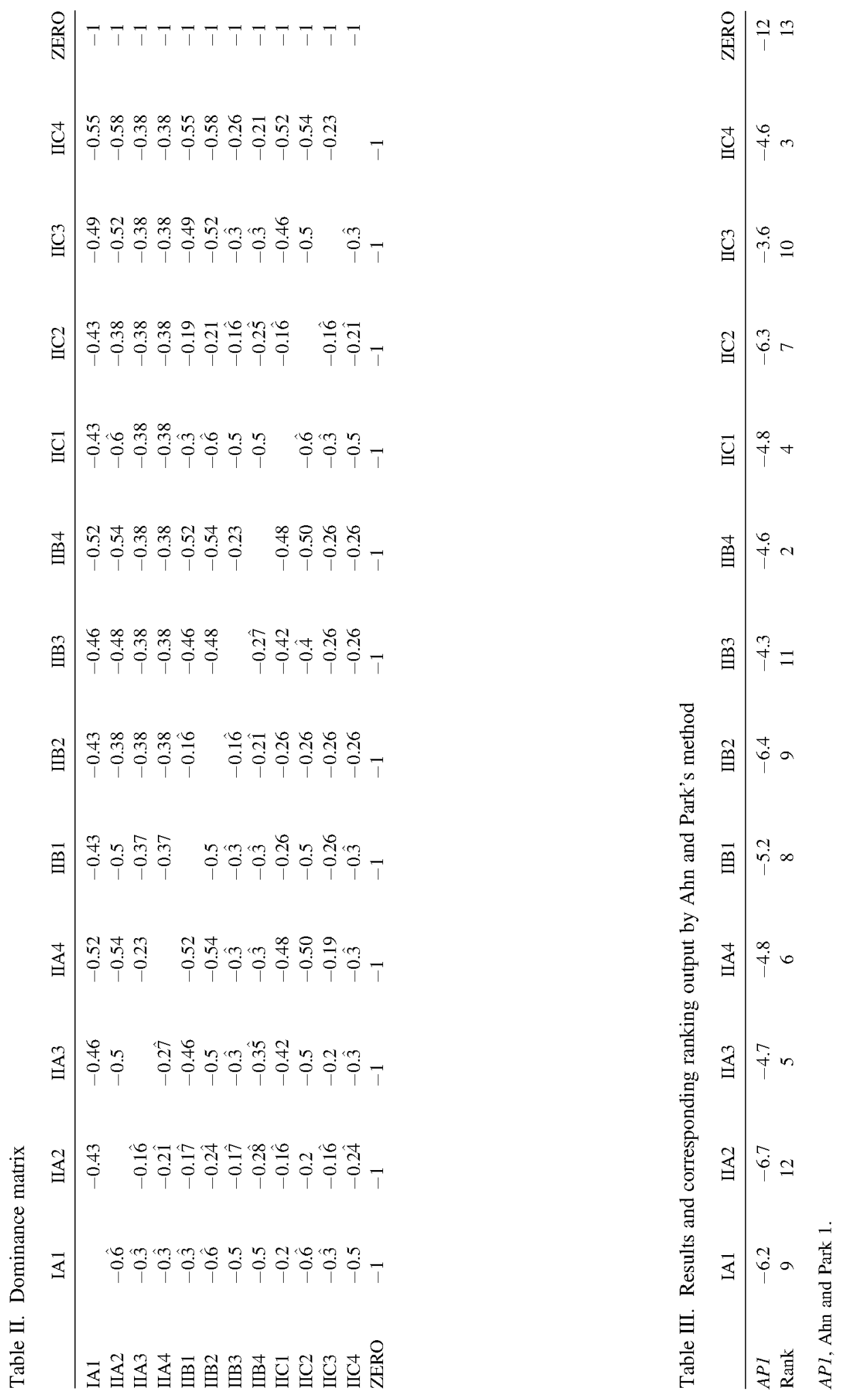


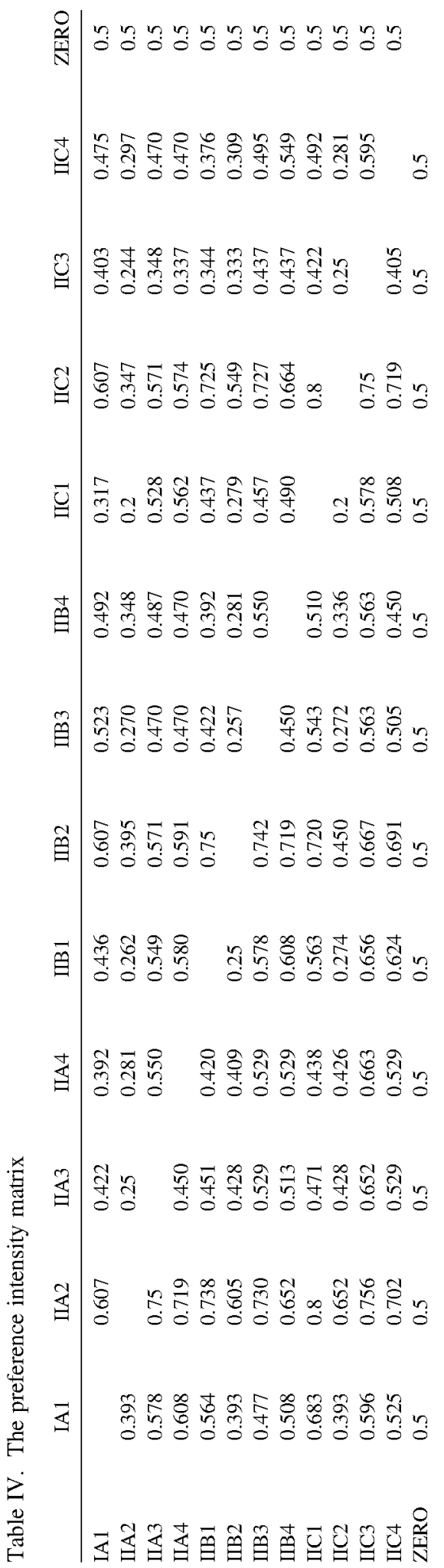

section to analyse their performance. For a decisionmaking problem with $m$ alternatives and $n$ attributes, the process would be as follows:

1. Randomly generate component utilities for each alternative in each attribute from a uniform distribution in $[0,1]$, leading to an $m \times n$ matrix. Normalize the columns in this matrix to make the smallest value 0 and the largest 1 and remove dominated alternatives.

2. Generate attribute weights representing their relative importance. Note that these weights are the TRUE weights, and the derived ranking of alternatives will be denoted as the TRUE ranking. To generate the TRUE weights, we first select $n-1$ independent random numbers from a uniform distribution on $(0,1)$ and then rank these numbers. Suppose the ranked numbers are $1 \geq r_{n-1} \geq \ldots \geq r_{2} \geq r_{1}>0$. The differences between adjacently ranked numbers are then used as the desired weights: $w_{n}^{T}=1-r_{n-1}$, $w_{n-1}^{T}=r_{n-1}-r_{n-2}, \ldots, w_{1}^{T}=r_{1}$. The resulting weights will sum 1 and be uniformly distributed in the weight space.

3. To derive the corresponding weight intervals, add and subtract the same quantity to precise values, leading to the lower and upper endpoints of the weight intervals. We used the quantities, $q$, of $0.1 /$ $2,0.2 / 2,0.3 / 2,0.4 / 2$ and $0.5 / 2$ that represent $10 \%$, $20 \%, 30 \%, 40 \%$ and $50 \%$ imprecision, respectively. In other words, $\left[w_{i}^{L}, w_{i}^{U}\right]=\left[w_{i}^{T}-q, w_{i}^{T}+q\right]$. If $w_{i}^{T}-q<0$, then $w_{i}^{T}-q=0$, and if $w_{i}^{T}+q>1$, then $w_{i}^{T}+q=1$ is considered. Throughout the simulation process, weights will be randomly generated from these weight intervals, $\left[w_{i}^{T}-q, w_{i}^{T}+q\right]$.

4. Compute the ranking of alternatives for each method according to their procedures and compare with the TRUE ranking, computed in step 2 . We use two measures of efficacy, hit ratio and rank-order correlation (Ahn and Park, 2008; Barron and Barrett, 1996; Winkler and Hays, 1985). The hit ratio is the proportion of all cases in which the method selects the same best alternative as in the TRUE ranking. Rank-order correlation represents how similar the overall alternative-ranking structures are in the TRUE and the method-driven rankings. It is calculated using Kendall's $\tau$ (Mateos et al., 2009): $\tau=1-2 \times$ (number of pairwise preference violations)/(total number of pair preferences).

Following (Ahn and Park, 2008; Mateos et al., 2010; Winkler and Hays, 1985), four different levels of alternatives $(m=3,5,7,10)$ and five different levels of 
Table V. Results and corresponding ranking output by DME2 method

\begin{tabular}{lcclllllllllll} 
& IA1 & IA2 & IIA3 & IIA4 & IIB1 & IIB2 & IIB3 & IIB4 & IIC1 & IIC2 & IIC3 & IIC4 & ZERO \\
\hline DME2 & 5.7 & 3.79 & 6.37 & 6.33 & 6.12 & 4.60 & 6.75 & 6.62 & 6.94 & 4.46 & 7.54 & 6.69 & 6 \\
Rank & 10 & 13 & 6 & 7 & 8 & 11 & 3 & 5 & 2 & 12 & 1 & 4 & 9
\end{tabular}

$D M E 2$, dominance measuring extension 2.

Table VI. SMAA and SMAA-2 results

\begin{tabular}{|c|c|c|c|c|c|c|c|c|}
\hline & $a^{1}$ & $a^{2}$ & $a^{3}$ & Holistic $a$ & $a^{1}$-rank & $a^{2}-\operatorname{rank}$ & $a^{3}$-rank & Holistic rank \\
\hline IA 1 & 5 & 11 & 18 & 28 & 8 & 7 & 8 & 9 \\
\hline IIA2 & 0 & 0 & 0 & 7 & 13 & 13 & 13 & 13 \\
\hline IIA3 & 5 & 12 & 22 & 34 & 7 & 6 & 6 & 6 \\
\hline IIA4 & 3 & 9 & 17 & 30 & 10 & 10 & 9 & 8 \\
\hline IIB 1 & 4 & 11 & 20 & 33 & 9 & 8 & 7 & 7 \\
\hline IIB2 & 0 & 1 & 2 & 14 & 11 & 11 & 11 & 12 \\
\hline IIB3 & 9 & 21 & 34 & 42 & 5 & 4 & 4 & 3 \\
\hline IIB 4 & 8 & 18 & 29 & 39 & 6 & 5 & 5 & 5 \\
\hline IIC1 & 18 & 33 & 45 & 49 & 2 & 2 & 2 & 2 \\
\hline $\mathrm{IIC} 2$ & 0 & 1 & 2 & 14 & 12 & 12 & 12 & 11 \\
\hline IIC3 & 27 & 48 & 64 & 61 & 1 & 1 & 1 & 1 \\
\hline IIC4 & 11 & 22 & 34 & 42 & 3 & 3 & 3 & 4 \\
\hline ZERO & 10 & 11 & 12 & 15 & 4 & 9 & 10 & 10 \\
\hline
\end{tabular}

$S M A A$, stochastic multicriteria acceptability analysis.

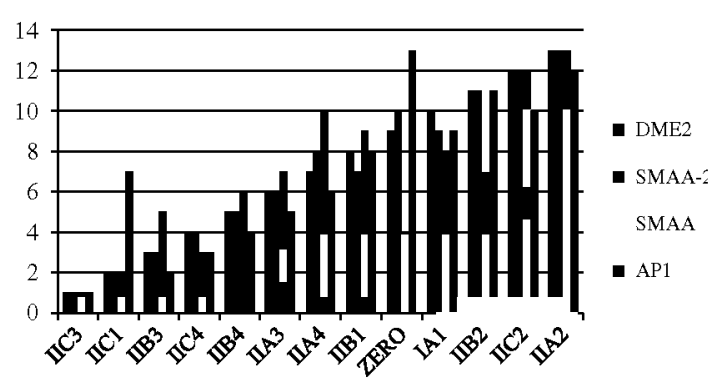

Figure 1. Rankings output by dominance measuring methods (Ahn and Park $1(A P 1)$ and dominance measuring extension $2(D M E 2)$ ) and the stochastic multicriteria acceptability analysis $(S M A A)$ and $S M A A-2$ methods.

attributes $(n=3,5,7,10,15)$ were considered to validate the output results. Also, 20000 trials were performed for each of the 20 design elements (alternatives $\times$ attributes).

The results of similar computational studies when ordinal information about weights is available are shown in Mateos et al. (2009). Surrogate weighting methods, methods that modified classical decision rules (REG, PES and OPT, see Section 2) and dominance measuring methods ( $A P 1, D M E 1, D M E 2)$ were considered. The findings were as follows:
1. Regarding methods that modified classical decision rules, the $R E G$ method appears to be better than the PES method. PES outperforms the OPT method. They are all outperformed by dominance measuring methods. Moreover, the results for DMEl and $D M E 2$ methods are similar, but both outperform AP1.

2. The average hit ratio decreases the more attributes there are for all the methods under consideration and any given number of alternatives. This decrease is more pronounced than if the number of alternatives is increased for the same number of attributes.

Surrogate weighting methods are also compared with dominance measuring methods when ordinal information about weights is available in Mateos et al. (2010). DME2 outperforms all the surrogate weighting methods except the $R O C$ method, which is the best in terms of the hit ratio and the rank-order correlation.

In the simulation study carried out in this work, we compare the $A P 1$ and $D M E 2$ methods with methods that analyse the set of favourable weights (SMAA and $S M A A-2$ methods). Additionally we use intervals rather than ordinal relations for weights. The $R O C$ method cannot be applied in this scenario. 
Figure 2 shows the average hit ratio for each of the 20 design elements when the interval length is 0.1 , that is, the average values of 20000 trials. The 20 design elements are represented on the abscissa, where the first value indicates the number of alternatives and the second the number of attributes.

The hit ratio decreases the more attributes there are for any given number of alternatives. Additionally, the number of alternatives also affects the hit ratio.

The DME2, SMAA and SMAA-2 methods clearly outperform the $A P 1$ method. On the other hand, though the performance of the DME2, SMAA and $S M A A-2$ methods is very similar, $S M A A$ and $S M A A-2$ slightly outperform $D M E 2$ for a small number of alternatives when the number of attributes is increased.

Looking at the rank-order correlation, see Figure 3, then

1. as the rank-order correlation decreases, the more attributes there are for any given number of alternatives. However, the number of alternatives does not affect the correlation.

2. the $S M A A-2$ method slightly outperforms the $D M E 2$ method, which also outperforms the AP1 method.

Note that SMAA was not considered when analysing the rank-order correlation because it is not always possible to derive a ranking of alternatives from this method.

The simulation was also run for the other listed percentages of imprecision (20\%, 30\%, 40\% and 50\%), and the results were similar, that is, the DME2, SMAA and $S M A A-2$ methods clearly outperform the $A P I$ method. DME2, SMAA and SMAA-2 output very

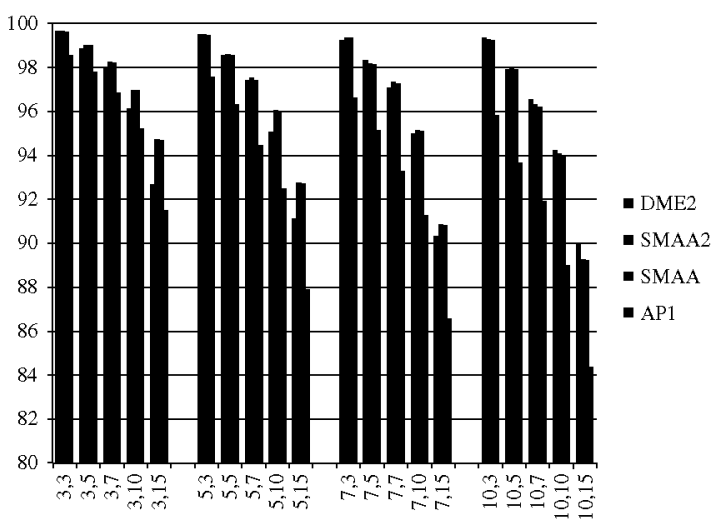

Figure 2. Hit ratio for the four methods. $D M E 2$, dominance measuring extension 2; SMAA, stochastic multicriteria acceptability analysis; $A P 1$, Ahn and Park 1.

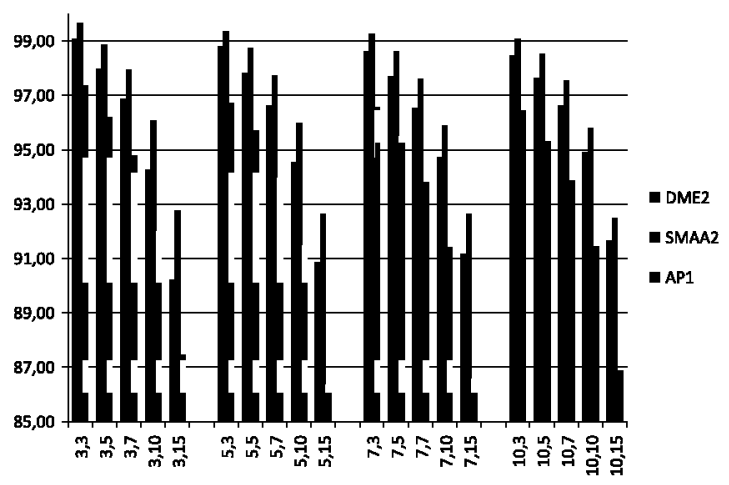

Figure 3. Rank-order correlation for the three methods. $D M E 2$, dominance measuring extension 2; SMAA, stochastic multicriteria acceptability analysis; $A P 1$, Ahn and Park 1.

similar results in terms of hit ratio, but SMAA-2 slightly outperforms $D M E 2$ on rank-order correlation.

\section{WEIGHTS FITTING INDEPENDENT NORMAL DISTRIBUTIONS OR WEIGHTS REPRESENTED BY FUZZY NUMBERS}

Now, we consider that the uncertainty about weights is represented by independent normal distributions with means $\left(\mu_{1}, \ldots, \mu_{n}\right)$ and variances $\left(\sigma_{1}^{2}, \cdots, \sigma_{n}^{2}\right)$, that is, $\left(w_{i} \sim N\left(\mu_{i}, \sigma_{i}^{2}\right), i=1, \ldots, n\right)$.

In this case, problem (2) can be represented by

$$
D_{k l}=\sum_{i=1}^{n} w_{i} z_{i}^{k l}
$$

s.t.

$$
w_{i} \sim N\left(\mu_{i}, \sigma_{i}^{2}\right), i=1, \ldots, n .
$$

Our aim is to take advantage of normal distribution properties, such as closure under linear combinations. For example, any linear combination of a number of independent normal distributions also follows a normal distribution. Therefore, it is well known that if $w_{i} \sim N\left(\mu_{i}, \sigma_{i}^{2}\right), i=1, \ldots, n$, then $D_{k l}$ has a normal distribution with mean $\sum_{i=1}^{n} z_{i}^{k l} \mu_{i}$ and variance $\sum_{i=1}^{n}\left(z_{i}^{k l}\right)^{2} \sigma_{i}^{2}$ because $D_{k l}$ is a linear combination of $w_{i}$.

The approach is based on the same idea as method $D M E 2$, where only weight intervals are considered to represent weights.

The new approach, denoted by DME2-Normal, can be implemented as follows. In the first step, the method computes the optimal solution of the optimization problems (3) for each pair of alternatives $A_{k}$ and $A_{l}$ 
$(k, l=1, \ldots, m)$. In the second step, the intensity with which alternative $A_{k}$ to is preferred to the others is computed. In the third step, a preference intensity measure for each alternative $A_{k}$ is computed. This measure represents a preference intensity measure of $A_{k}$. The third step ranks the alternatives considering the preference intensity measure. Alternative $A_{k}$ is better than alternative $A_{l}$ if the preference intensity measure of alternative $A_{k}$ is greater than the preference intensity measure of alternative $A_{l}$.

1. Compute $z_{i}^{k l}$ for alternatives $A_{k}$ and $A_{l}(k, l=1, \ldots, m)$ and each attribute $X_{i}(i=1, \ldots, n)$ following (3).

2. Compute

$$
P I_{k l}=\int_{0}^{\infty} f_{k l}(x) d x
$$

where $f_{k l}(x)$ is the density function of the variable $D_{k l}$, that is, $f_{k l}(x)$ is the density function of a normal distribution variable with mean $\sum_{i=1}^{n} z_{i}^{k l} \mu_{i}$ and variance $\sum_{i=1}^{n}\left(z_{i}^{k l}\right)^{2} \sigma_{i}^{2}$.

3. Compute a preference intensity measure for each alternative $A_{k}$ :

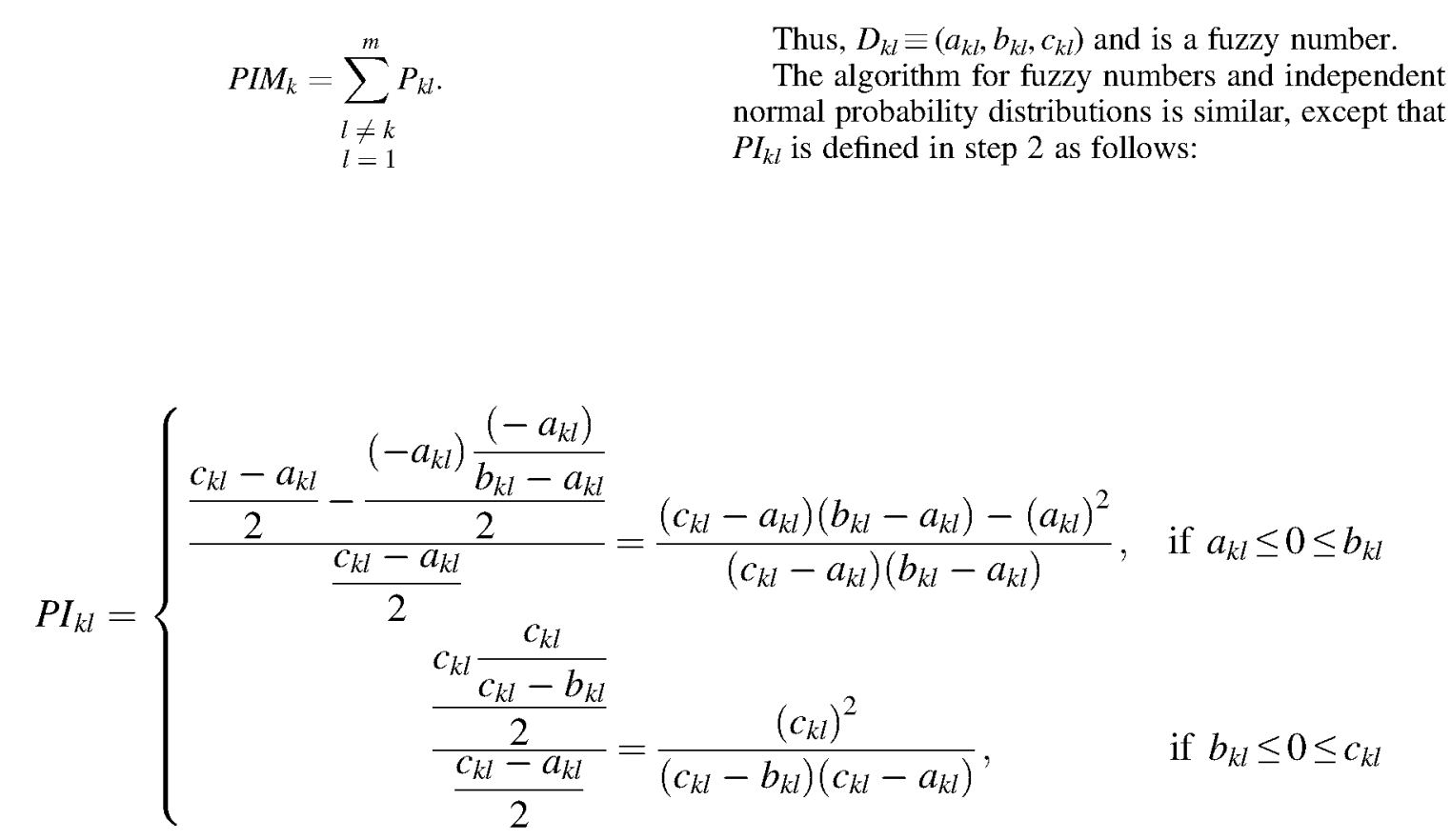
where the best (rank 1) is the alternative with the greatest $P I M_{k}$ and the worst is the alternative with the least $P I M_{k}$.

If the weights are represented by triangular or trapezoidal fuzzy numbers, adaptation is direct because the product of a scalar multiplied by a triangular or trapezoidal fuzzy number and the sum of triangular or trapezoidal fuzzy numbers is another triangular or trapezoidal fuzzy number. For example, if weights $w_{i}$ are represented by triangular fuzzy numbers $\left(a_{i}, b_{i}, c_{i}\right), \forall i=1$, $\ldots, n$, then problem (5) is

$$
\begin{array}{ll} 
& D_{k l}=\sum_{i=1}^{n} w_{i} z_{i}^{k l} \\
\text { s.t. } \quad & w_{i} \equiv\left(a_{i}, b_{i}, c_{i}\right), i=1, \ldots, n .
\end{array}
$$

To compute the components of $D_{k l}$, we must take into account that

$$
w_{i} z_{i}^{k l} \equiv\left\{\begin{array}{l}
\left(a_{i} z_{i}^{k l}, b_{i} z_{i}^{k l}, c_{i} z_{i}^{k l}\right), \text { if } z_{i}^{k l}>0 \\
\left(c_{i} z_{i}^{k l}, b_{i} z_{i}^{k l}, a_{i} z_{i}^{k l}\right), \text { if } z_{i}^{k l}<0
\end{array}\right.
$$

and

$$
\left(a_{i}, b_{i}, c_{i}\right)+\left(a_{j}, b_{j}, c_{j}\right)=\left(a_{i}+a_{j}, b_{i}+b_{j}, c_{i}+c_{j}\right) .
$$




\section{CONCLUSIONS}

In real complex decision-making problems, it is not easy to elicit precise values for the weights representing the relative importance of criteria, which often leads to information being incomplete. A recent approach for dealing with such situations is to use information about each alternative's intensity of dominance employing what are known as dominance measuring methods.

We have reviewed the features of existing dominance measuring methods, all applied when there are ordinal relations regarding attribute weights, that is, DMs ranked attributes in descending order of importance. Simulation studies have been conducted in this scenario to compare the methods with each other and with other approaches, such as surrogate weighting methods and modified decision rules. The results showed that DME2 performs better than other dominance measuring methods and the adaptation of classical decision rules and comes quite close to the $R O C$ method, which was identified as the best approach.

However, incomplete information about weights can be expressed in other ways, for example, by weight intervals fitting independent normal probability distributions or represented by triangular or trapezoidal fuzzy numbers. Moreover, the $R O C$ method cannot be applied in these situations.

A simulation study has been run on the aforementioned scenarios to analyse the DME2 method, and again, compare it with the methods suggested by Ahn and Park, with modified decision rules and with $S M A A$ and $S M A A-2$ methods.

The results show that DME2 and SMAA-2 outperform the other methods in terms of the identification of the best alternative and the overall ranking of alternatives. Different situations of imprecision were analysed $(10 \%, 20 \%, 30 \%, 40 \%$ and $50 \%$ imprecision), leading to the same conclusion. Furthermore, all these methods are sensitive to the number of attributes. An increase in the number of attributes implies the reduction of the proportion of well-ordered alternatives. On the other hand, the increase in the number of alternatives does not significantly affect the goodness of the method.

Although SMAA-2 slightly outperforms DME2, $D M E 2$ can be used when incomplete information about weights is expressed not just as weight intervals but also as weights satisfying linear or non-linear constraints, weights represented by fuzzy numbers or weights fitting normal probability distributions. However, the SMAA-2 method cannot consider fuzzy numbers, and simulation techniques could be hard to apply considering constraints on weights.

\section{REFERENCES}

Ahn BS. 2003. Extending Malakooti's model for ranking multicriteria alternatives with preference strength and partial information. IEEE Transactions on Systems, Management and Cybernetics, Part A: Systems and Humans 33(3): 281-287.

Ahn BS, Park KS. 2008. Comparing methods for multiattribute decision making with ordinal weights. Computers and Operations Research 35: 1660-1670.

Bana e Costa CA. 1986. A multicriteria decision aid methodology to deal with conflicting situations on the weights. European Journal of Operational Research 26: 22-34.

Barron F, Barrett B. 1996. Decision quality using ranked attribute weights. Management Science 42(11): 1515-1523.

Charnetski JR, Soland RM. 1978. Multiple-attribute decision making with partial information: the comparative hypervolume criterion. Naval Research Logistics Quaterly 25: 279-288.

Eum Y, Park KS, Kim H. 2001. Establishing dominance and potential optimality in multi-criteria analysis with imprecise weights and values. Computers and Operations Research 28(5): 397-409.

Hokkanen J, Lahdelma R, Maiettinen K, Salminen P. 1999. A multiple criteria decision model for analyzing and choosing among different development patterns for the Helsinki cargo harbor. Socio-Economic Planning Sciences 33: 1-23.

Jiménez A, Mateos A, Ríos-Insua S. 2005. Monte Carlo simulation techniques in a decision support system for group decision-making. Group Decision and Negotiation 14(2): 109-130.

Keeney RL, Raiffa H. 1976. Decision with Multiple Objectives: Preferences and Value-Tradeoffs. Wiley: New York.

Kirkwood CW, Corner JL. 1993. The effectiveness of partial information about attribute weights for ranking alternatives in multiattribute decision making. Organization Behavior and Human Decision Processes 54: 456-476.

Lahdelma R, Salminen P. 1998. SMAA-stochastic multiobjective acceptability analysis. European Journal of Operational Research 106: 137-143.

Lahdelma R, Salminen P. 2001. SMAA2 - stochastic multiobjective acceptability analysis for group decision making. Operational Research 49: 444454.

Lee K, Park KS, Kim H. 2002. Dominance, potential optimality, imprecise information, and hierarchical structure in multi-criteria analysis. Computers and Operations Research 29: 1267-1281. 
Malakooti B. 2000. Ranking and screening multiple criteria alternatives with partial information and use of ordinal and cardinal strength of preferences. IEEE Transactions on Systems, Management and Cybernetics: Part A 30(3): 787-801.

Mateos A, Jiménez A, Ríos-Insua S. 2006. Monte Carlo simulation techniques for group decision-making with incomplete information. European Journal of Operations Research 174(3): 1842-1864.

Mateos A, Ríos-Insua S, Jiménez A. 2007. Dominance, potential optimality and alternative ranking in imprecise decision making. Joumal of the Operational Research Society 58(3): 326-336.

Mateos A, Jiménez A, Blanco JF. 2009. Ranking Methods Based on Dominance Measures Accounting for Imprecision. Algorithmic Decision Theory. LNAI 5783. Springer: Berlin; 328-339.

Mateos A, Jiménez A, Blanco JF. 2010. A MCDM Ranking Method Based on a Dominance Measure: Computational Study, Group Decision and Negotiation (in revision).

Nijkamp P, Rietveld P, Voogd H. 1990. Multicriteria Evaluation Physical Planning. North Holland: Amsterdam.

Park K. 2004. Mathematical programming models for characterizing dominance and potential optimality when multicriteria alternative values and weights are simultaneously incomplete. IEEE Transactions on Systems, Management and Cybemetics, Part A: Systems and Humans 34: 601-614.

Puerto J, Marmol AM, Monroy L, Fernández FR. 2000. Decision criteria with partial information. International Transactions in Operational Research 7: 51-65.
Raiffa H. 1982. The Art and Science of Negotiation. Harvard University Press: Cambridge.

Sage A, White CC. 1984. Ariadne: a knowledge-based interactive system for planning and decision support. IEEE Transactions on Systems, Management and Cybernetics, Part A: Systems and Humans 14: 35-47.

Salo A, Hämäläinen RP. 2001. Preference ratio in multiattribute evaluation (PRIME) elicitation and decision procedures under incomplete information. IEEE Transactions on Systems, Management and Cybernetics: Part A 31(6): 533-545.

Sarabando P, Dias LC. 2009. Multi-attribute choice with ordinal information: a comparison of different decision rules. IEEE Transactions on Systems, Management and Cybernetics, Part A 39(3): 545-554.

Sarabando P, Dias LC. 2010. Simple Procedures of Choice in Multicriteria Problems without Precise Information about the Alternatives Values. Computers and Operations Research 37(12): 2239-2247.

Stewart TJ. 1996. Robustness of additive value function method in MCDM. Journal of Multi-Criteria Decision Analysis 5: 301-309.

Voogd H. 1983. Multicriteria Evaluation for Urban and Regional Planning. Pion Ltd.: London.

Weber M. 1987. Decision making with incomplete information. European Journal of Operational Research 28: 44-57.

Winkler RL, Hays WL. 1985. Statistics: Probability, Inference and Decision. Holt, Rinehart \& Winston: New York. 